\title{
Akteure von Internet Governance
}

\subsection{Analytische Zugänge zu Akteuren}

Wie für alle gesellschaftlichen Bereiche oder - systemtheoretisch - für alle Teilsysteme lässt sich die Analyse von Internet Governance primär als Frage des objektbezogenen, interessengeleiteten und zielorientierten Handelns begreifen: mithin als die analytische Suche nach den relevanten Akteuren, ihren strukturellen Kontexten, den Zielen und Reichweiten ihres Handelns, den Ebenen ihrer Aktionen, den Formen ihrer Organisation und Institutionalisierung sowie nach ihrer Interessengebundenheit und gesellschaftlichen Repräsentativität, dem Cui bono. Allerdings lassen sich bei einem derart dynamischen, sich schnell veränderndem und dementsprechend nicht fixierbaren Gegenstandsfeld wie dem Internet diese Fragen zu keinem Zeitpunkt umfassend und erschöpfend behandeln, sondern die Befunde sind allenfalls zeitbezogene Zwischenübersichten, vielfach auch nur heuristische Sondierungen in sich jeweils neu konstituierenden Handlungsfeldern: Denn zum einen ist das Internet trotz seiner nunmehr 30-jährigen Entwicklung noch so neu, dass etablierte Akteure die für sie relevanten Regelungsbedarfe und Interessenspotentiale vielfach noch suchen bzw. mit den rapiden Entwicklungen ständig neu definieren müssen. Zum anderen entstehen unentwegt neue Gruppierungen und Akteure - zumal in zivilgesellschaftlichen Segmenten -, die ihre Einfluss- und Interessenspotentiale für das Internet erst definieren und begründen müssen. Und schließlich stellt das Internet selbst viele bis zum 20. Jahrhundert gewachsene und konsolidierte Strukturen und gesellschaftliche Mechanismen zusammen mit anderen globalen Trends wie der Globalisierung, dem Wandel und der Verflechtung von Verkehr, Wirtschaft und Handel, den Vergesellschaftungs- und Verrechtlichungsprozessen jenseits des Staates (Neyer 2004; Meyers 2011, 492), den intensivierenden Kommunikationsbeziehungen und 
Medialisierungsschüben grundsätzlich in Frage (Hepp u. a. 2005; Schweiger und Beck 2010), so dass sich Aktionsfelder für das Handeln ständig neu aufdrängen oder ergeben.

Markantestes Beispiel ist der Bestand und der Souveränitätsanspruch des im 19. Jahrhundert fixierten Nationalstaates. Ihn sehen etliche sozialwissenschaftliche Beobachter zunehmend erodieren, mindestens viele seiner Kompetenzen verlustig gehen oder - positiver - in supra- und transnationale Konstrukten aufgehen (Zürn 1998; Leibfried und Zürn 2006; Schneckener 2006). Dagegen wehren sich die Vertreter und Profiteure der Nationalstaatlichkeit vehement, auch mit massiven Restriktionen, insbesondere die Repräsentanten autoritärer Regime. Insofern werden einerseits anhand der Regelung des Internets, der Internet Governance, stellvertretende Konflikte und Okkupationsambitionen ausgetragen, die letztlich ungleich weiter und tiefer sind als die konkreten Internet-Regeln. Zum anderen wurde und wird das Internet wiederholt dafür ausgelobt, dass seine nonkonformistische Nutzung rigide und repressive Staatlichkeit unterhöhlt und für rebellierende Bewegungen mehr Einfluss, Transparenz und Freiheit, letztlich Demokratisierung ermöglicht. So wurden die Proteste und Revolten etwa in den arabischen Ländern der letzten Jahre wiederholt als von Handys, Skype, i-Pads und sozialen Netzwerken geförderte Revolutionen charakterisiert (Schröder 2012).

Beispielhaft lassen sich die Erosionen am Begriff des Politischen beobachten: Ließ sich Politik in den 1990er Jahren noch umfassend als „für alle die Probleme zuständig [definieren], die von den anderen Teilsystemen nicht gelöst werden und dem politischen System als Problemadressat attribuiert werden", weshalb sie „zumindest innerhalb bestimmter territorialer Grenzen“ und kraft der „Monopolisierung legitimer Gewalt in der Hand des Staates“ die einzigartige Kompetenz besitzt, „die Rahmenbedingungen der anderen Systeme primär durch Entscheidungen \{zu setzen], die im Konfliktfall verbindlich durchsetzbar sind“ (Gerhardt und Neidhardt 1990, 8f), so dürfte eine solche exklusive Definition von Politik heute kaum mehr valide und akzeptabel sein - es sei denn, man überdehnt den herkömmlichen Begriff des Politischen auf alles soziale, tendenziell allgemein verbindliche Handeln, wie es allerdings im Governance-Begriff angelegt ist. Etliche Forschungsprojekte haben sich in jüngster Zeit gerade um die durch diverse Faktoren verursachten Transformationen des Staatlichen bzw. Politischen und um die Aufweichungen zwischen Öffentlichkeit und Privatheit gekümmert (Sennett 1983; Faulstich und Hickethier 2000; Leibfried und Zürn 2006; Benz u. a. 2007; Neuberger 2009; Emmer und Wolling 2010).

Allerdings wurden dabei die Funktion bzw. die Wirkmacht des Internets für bzw. auf diese Prozesse bislang nicht eingehend untersucht, oder die verfügbaren Studien kommen zu kontroversen Befunden (Brand 2012, 81ff): Die einen 
erkennen auch beim Internet den in der sozial- und kommunikationswissenschaftlichen Forschung schon häufig konstatierten, ursprünglich von dem amerikanischen Soziologen Robert K Merten (1985) thematisierten, so genannten „Matthäus-Effekt“ („Wer hat, dem wird gegeben“) erneut bestätigt; will heißen: nur die gebildeten, sozial einigermaßen erfolgreichen, kosmopolitisch ausgerichteten und kommunikationstechnisch versierten Akteure können mit dem Internet sozialen Wandel und Transformationen der gesellschaftlichen Strukturen anstoßen (Marr und Zilien 2010, 274). Mithin setzt das Internet ein gewisses Niveau der technischen und sozialen Entwicklung in doppelter Hinsicht voraus: Diese Voraussetzungen gelten einerseits hinsichtlich der verfügbaren technischen, materiellen Infrastruktur und der finanziellen Ressourcen, so dass es überhaupt einigermaßen breit eingeführt und von Individuen und Gruppen für die jeweils eigenen Interessen und Absichten genutzt werden kann. Die individuellen Fähigkeiten werden im größeren Zusammenhang als Medienkompetenz (media literacy) oder spezielle Internetkompetenz (computer and information oder digital literacy) bezeichnet (Moser u. a. 2011). Allein schon die Beherrschung einer translokalen und -nationalen Sprache - in der Regel: das Englische - ist vielfach eine kaum überwindbare Barriere.

Andererseits wird das Internet systemwidrig in territoriale und nationale Grenzen eingezwängt und fungiert als Herrschaftsinstrument wie alle anderen Massenmedien. Dann sind vorrangigen Funktionen Propaganda und Loyalitätssicherung für das herrschende Regime. Unter solchen Bedingungen werden daher von eher technologieskeptischen und am Status quo orientierten Positionen dem Internet kaum transformierende Potentiale und kohäsive Effekte in globaler Dimension eingeräumt; vielmehr werden eher Vertiefungen der sozialen, wirtschaftlichen, politisch-ideologischen und kommunikativen Spaltungen und Verschärfungen der weltweiten Desintegration befürchtet (Wischermannu und Thomas 2008; Zilien 2009; Stegbauer 2012).

Um analytisch aufzuarbeiten, welche Akteure in welchen Handlungsfeldern von Internet mit welchen Strategien, Interessen und Erfolgsaussichten tätig waren und sind, befasst sich Kap. 5 zunächst eher deskriptiv mit den maßgeblichen Akteuren, wobei es sich nur um eine exemplarische Auswahl und um eine eher kursorische Porträtierung ihrer angestammten Aufgaben- und Tätigkeitsfelder, die nun auf Internet Governance übertragen oder von ihr erweitert werden, oder ihrer neu ergriffenen Internet-Aktivitäten handeln kann. Sodann folgen in Kap. 6 die Aufarbeitung, Darstellung und Einordung signifikanter Handlungsfelder von Internet Governance, wie sie sich in den letzten Jahrzehnten herausgebildet haben und auch in den aktuellen Diskussionen darstellen.

Unter Akteure werden alle Institutionen, Organisationen, Gruppen und Personen verstanden, die ,an der Gestaltung von Politik bzw. an politischen Entscheidungen“ hier: hinsichtlich des Internets in seiner besagten Breite und Vielseitigkeit - beteiligt 
sind oder sein wollen (Emmer und Bräuer 2010, 312). Diese Einflussnahmen können unmittelbar aufgrund verfügbarer oder übertragener Entscheidungskompetenzen oder indirekt über die Steuerung von Öffentlichkeit und Medien erfolgen - wobei diese immens komplexen Entscheidungsabfolgen gerade infolge der sich ständig intensivierenden Medialisierungsprozesse enorm zunehmen. Zirkuläre Potenzierungsund Eskalierungsfaktoren sind hier mächtig am Werk. Herkömmlicherweise lassen sich öffentliche bzw. staatliche und private Akteure unterscheiden, etwa anhand des Modus ihrer Legitimierung und Institutionalisierung, der Allgemeinheit bzw. Generalisierungsfähigkeit ihrer Interessen und Ziele („Gemeinwohl“) und der (repräsentativen) Bestellung und Vertretung ihrer Repräsentanten. Im nationalen Kontext sind diese Charakteristika noch weitgehend intakt und anerkannt; auf supra- und internationaler und erst recht auf globaler Ebene sind sie allenfalls noch eingeschränkt gültig und mischen sich vielseitig. So ringen etwa Europäisches Parlament und Europarat mit der Europäischen Kommission und dem Europäischen Rat der Nationalregierungen schon lange um ihre Kompetenzen und Legitimation. International finden sich noch viele andere Misch- und Rudimentärformen oder nur partiell legitimierte Organisationen, wie zu zeigen sein wird.

Gerade die Entstehung und Verbreitung der so genannten Zivilgesellschaft verdanken der schwindenden Legitimität und Entscheidungskraft des staatlichen, formellen Handelns und der Formierungen über- bzw. zwischenstattlicher Handlungsbedarfe, die transnationale, meist globale Risiken und Gefahrenherde wie die Luftverschmutzung, die Erderwärmung, der Klimawandel, die anhaltende Zerstörung sämtlicher natürlicher Ressourcen, aber auch transnationale Entwicklungen wie die Finanz- und Handelsprozesse, die Energieprobleme, die Kriegsgefahren etc. heraufbeschwören, ihre wachsende Legitimität, und sie steigern die politischen Einflusspotentiale des so genannten dritten Sektors zwischen Staatlichkeit - früher auch Öffentlichkeit - und Privatheit. Die anhaltenden Medialisierungen führen dazu, dass kaum ein einigermaßen gesellschaftlich relevantes oder auch nur vermeintlich relevantes Handeln auch von privaten Akteuren auf Dauer geheim bleibt, so dass sich Risiken der Veröffentlichung ständig ergeben. Allerdings werden auch viele - letztlich unbedeutende - Ereignisse und Personen $\mathrm{zu}$ angeblich öffentlichen Sujets von den Medien, den professionellen wie den amateurhaften, eigentlich ,privaten' Publizisten hochgejubelt, so dass sich die überkommenen Terrains und Grenzen zwischen öffentlich und privat unentwegt mischen. Einigermaßen wahrgenommener und einflussreicher Akteur in der medialen Öffentlichkeit kann inzwischen jede/ $r$ und alles werden (Pörksen und Detel 2012).

Insofern sind angestammte Konditionalaussagen wie „Voraussetzungen für [die] Handlungsfähigkeit [von Akteuren] ist die Verfügung über Ressourcen und Handlungsorientierungen wie politische Ziele oder Programme“ (Emmer und 
Bräuer 2010, 312) allenfalls noch partiell gültig - oder der Begriff der Ressource muss auf symbolische und kommunikative ausgedehnt werden, die in einer ,Mediengesellschaft' mindestens so wichtig und effektiv sind wie materielle. Auch die Beanspruchung von „Zielen und Programmen“ nimmt ständig ab; selbst die etablierten Parteien als die politischen Akteure per se verstecken sich mehr und mehr hinter nur noch lapidaren, pauschalen Ziel- und Programmdeklamationen oder auch hinter Ziel- und Programmlosigkeit -, um für möglichst alle Interessenund Klientelgruppen unter den Wählern wählbar zu sein. Eher profilieren sich die zivilgesellschaftlichen Akteure durch dezidierte, meist aber spezielle Ziel- und programmatische Aussagen. Hingegen verfügen nur wenige von ihnen über ausreichende materielle und organisatorische Ressourcen, denn viele entstehen spontan und unorganisiert, anlässlich eines bestimmten Problems, gerade auch hinsichtlich des Internets (wie vor gut einem Jahr die kurz währenden ACTA-Bewegungen gegen das umstrittene Abkommen gegen Produktpiraterie), sie verfallen auch wieder oder reihen sich in die üblichen Bahnen des Politik- und Protestbetriebes ein. Diese Fluidität drückt sich in der Bezeichnung „Aktivisten“ aus, die sich so von den Akteuren unterscheiden (Ebd., 322ff; Wendelin und Löblich 2013).

Um die Vielzahl und Unterschiedlichkeit von Akteuren zu überschauen, werden diverse Typsierungen vorgeschlagen. Naheliegend ist die Unterscheidung nach Aktionsebenen, wobei die Lokalitäten der Akteure anders sein können als die der Ziele und Ansätze ihrer Aktionen: Dafür bieten sich die üblichen Einordnungen also global, supranational, international, regional - hier: besonders europäisch - und national an (Donges und Puppies 2010, 87ff). Als nächstes sollten die Art bzw. der Grad ihrer Institutionalisierung bzw. Organisation beschrieben werden. Hier stehen etwa Nationalstaaten oder ihre Bündnisse den so genannten NGOs diametral gegenüber; ebenso gehören zum Status und Profil eines Akteurs, wer Mitglied in seiner Organisation - etwa Staaten oder Privatpersonen, Verbände oder Unternehmen - ist, wie sie ihre Vertreter rekrutieren und in die jeweiligen Gremien schicken, wie ihre Legitimation erwirkt wird, wie sie kontrolliert werden und Rechenschaft ablegen. In Gesetzen, Satzungen und/oder Abkommen werden gemeinhin die Kompetenzen und Aufgaben der Akteure definiert, die entweder unabhängig vom Internet fixiert sind, darauf ausgeweitet und spezifiziert oder die allein im Hinblick auf Internet Governance formuliert und vollzogen werden.

Aus den gesetzlichen Konstellationen ergibt sich beispielsweise, ob Internet Governance eher oder ausschließlich als Selbst-(Ko-)Regulierung der Beteiligten oder als hierarchische Regulierung durch eine legitimierte, übergeordnete Instanz ausgeübt wird. Sicherlich lassen sich die Akteure auch danach klassifizieren, welche Art von Zielen und Aktivitäten sie für die Internet Governance verfolgen. Allerdings beziehen sich solche Dimensionen eher auf die Sujets und 
Aufgabenfelder, weniger auf die Akteure selbst; dennoch lassen sie sich kaum voneinander trennen. Eine Einteilung in "drei Schichten der Internet Governance“ (Ebd., 93f), nämlich in die "physisch[technisch]e Infrastruktur“, den "code layer oder logical infrastructure" (für Software und "für das Funktionieren des Internets notwendigen Protokolle“) sowie den „content layer" (für jegliche Inhalte und Materialien, die gespeichert oder übermittelt werden oder auf die zugegriffen wird), dürfte zu kurz greifen und nur partielle Aspekte abdecken. Denn gerade die umfassenden, momentan auch noch umstrittensten Themen, die über die gesamte Beschaffenheit, Funktionalität und Zukunft des Internets entscheiden und woran vor allem die globalen und internationalen Akteure vorrangiges Interesse haben wie etwa Netzneutralität, E-Commerce, Datenschutz, Copyright, passen nicht unter besagte Kategorien. Die jeweiligen Reichweiten der Aktionen lassen sich entweder normativ - als Sollensziele - oder empirisch-faktisch - als erreichte Ziele und Wirkungen - definieren. Beispielhaft können einzelne Aktionen vorgestellt werden, um an ihnen Funktionen, Vorgehensweisen und Effektivitäten der Handlungen zu analysieren und zu illustrieren.

Exemplarisch seien diese Dimensionen und Attribute (nach Donges und Puppis 2010, 87ff) in der Matrix in Tab. 5.1 dargestellt.

\subsection{Internetakteure im Einzelnen}

Mit der Diskussion um das Management und die Kontrolle über das Internet beschäftigt sich eine ganze Reihe nationaler, regionaler und internationaler Organisationen, privatwirtschaftlicher und zivilgesellschaftlicher Verbände. Es ist über die letzten beiden Jahrzehnte nicht gelungen, eine globale, von allen Staaten und der Zivilgesellschaft akzeptierte Internet-Architektur mit regelnder oder auch nur koordinierender Zuständigkeit für alle Felder der Internet-Governance aufzubauen. Vielmehr haben wir es in diesem Bereich mit stark fragmentierten Zuständigkeiten, Regelungsdefiziten, aber auch Kompetenzüberschneidungen $\mathrm{zu}$ tun, welche die tatsächlich existente Internet Governance unübersichtlich machen. Beispielsweise konnte sich ICANN nicht als global und allseits legitimierte Institution durchsetzen, besteht aber mangels institutioneller Alternative fort. Die um deren Kompetenzen konkurrierende und als Ersatz von den Entwicklungsländern und vormals sozialistischen Staaten favorisierte ITU wird von den wirtschaftlich mächtigen westlichen Staaten und Unternehmen mit Misstrauen betrachtet. Der mit großen Hoffnungen gestartete Weltinformationsgipfel der UNESCO versandete letztlich in seinem ständig tagenden, aber wenig bewegendem Appendix, genannt Internet Governance Forum. Der Einfluss der Nationalstaaten 


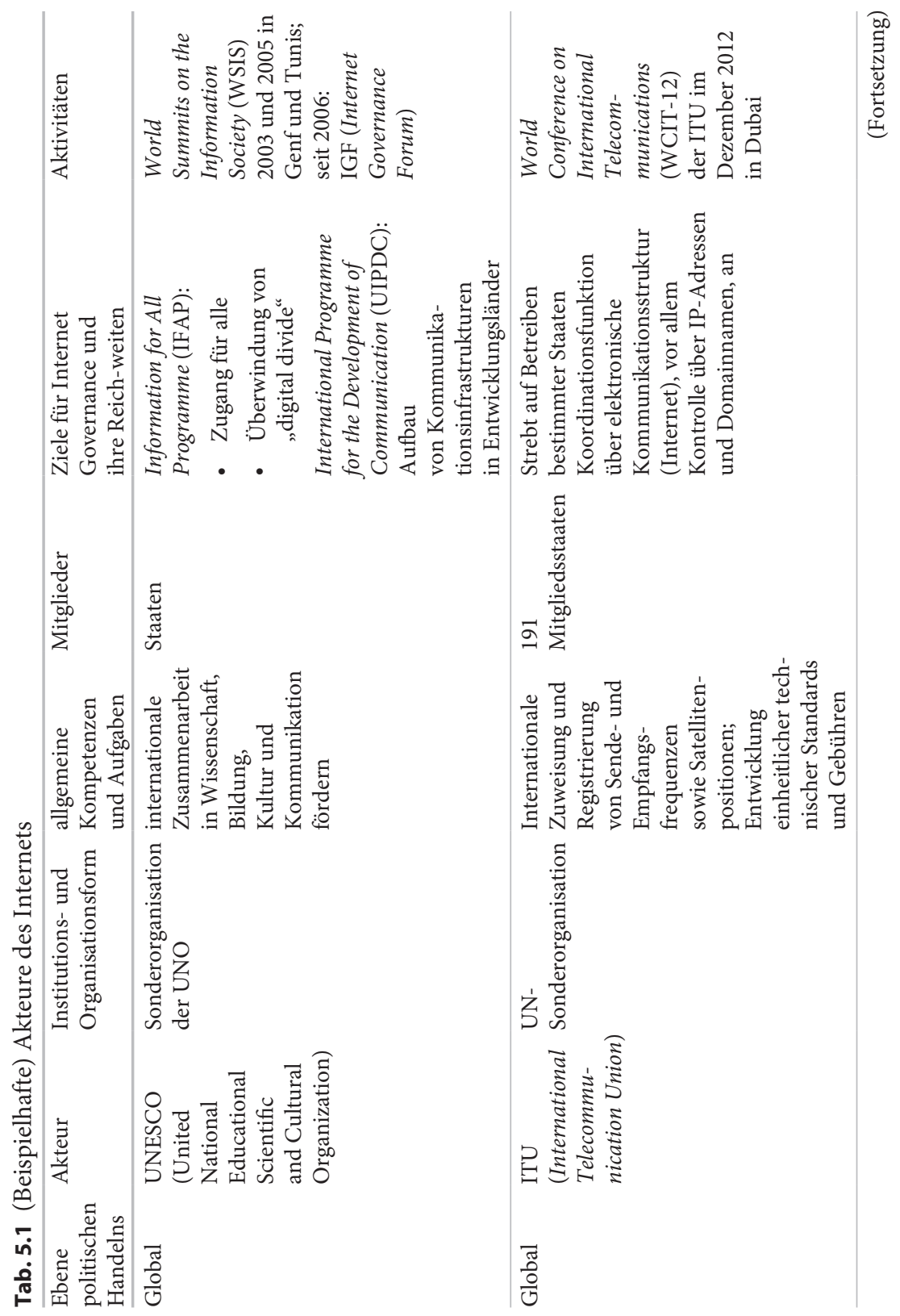




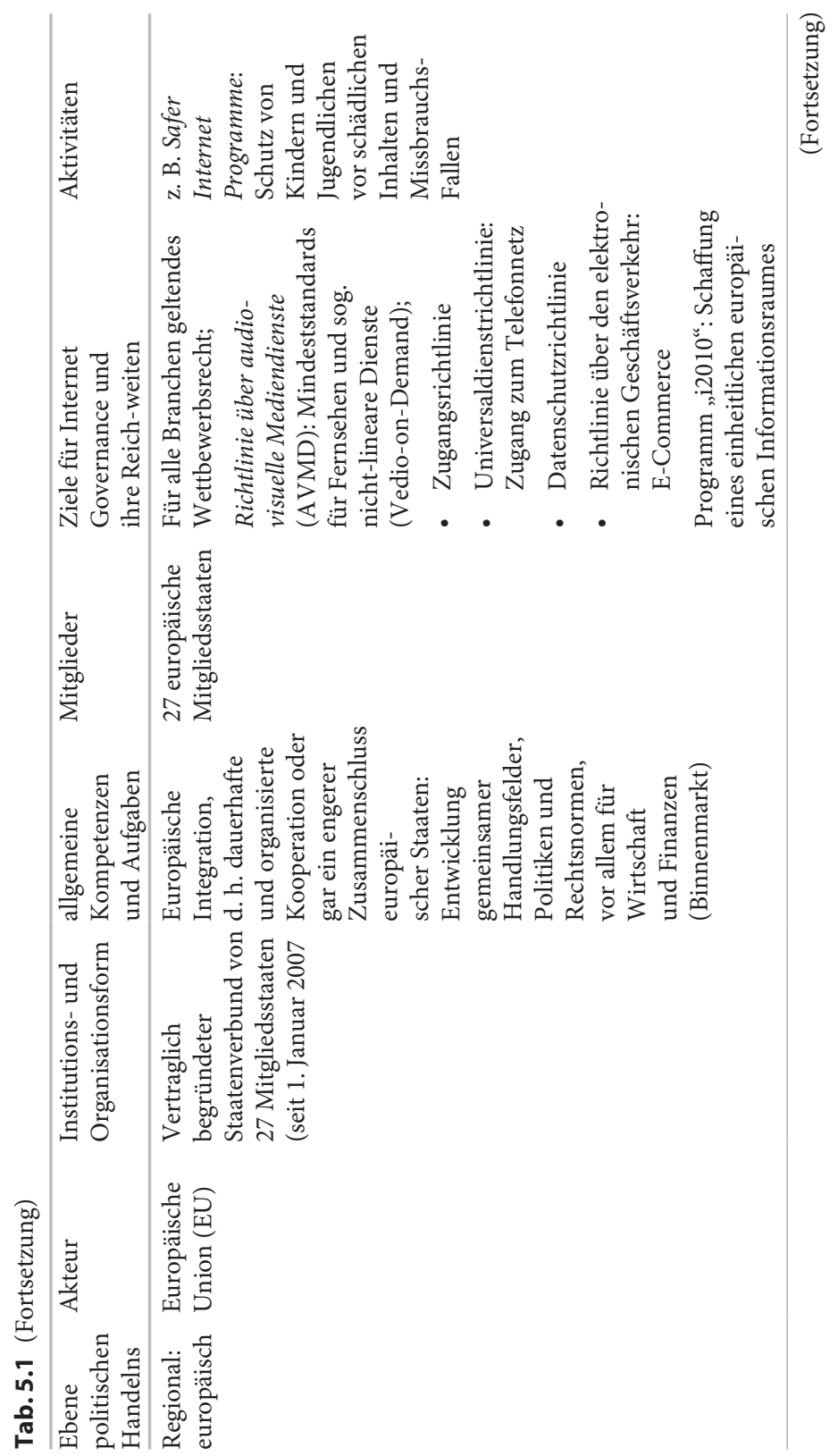




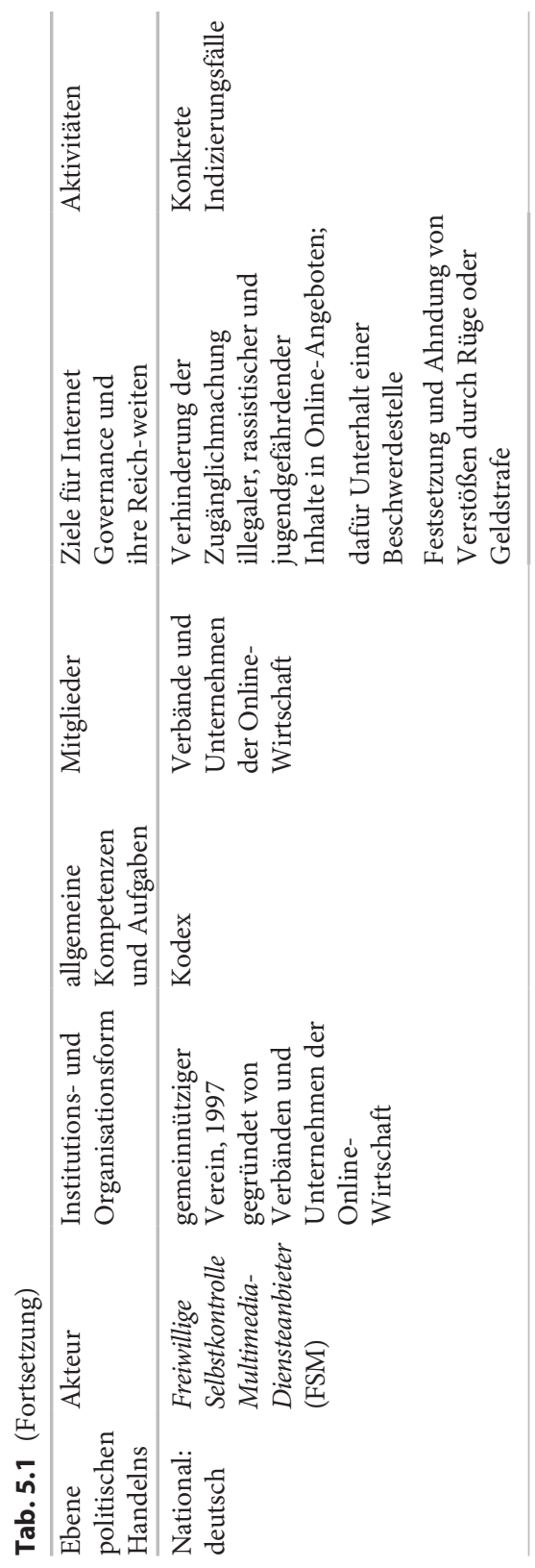


auf das angeblich so grenzenlose Internet wächst wieder, aber nicht so stark, dass die übrigen Stakeholder (Privatunternehmen, Nichtregierungsorganisationen) ausgeschaltet worden wären. Viele internationale und regionale Organisationen ringen um Einfluss auf das Netz und seine Regulierung, konnten sich aber nur partielle Bereiche davon sichern. Nationale Regierungen versuchen ihre Regelungskompetenzen eifersüchtig $\mathrm{zu}$ wahren, kommen aber an regionaler oder internationaler Abstimmung und Harmonisierung nicht mehr vorbei (ähnlich Mueller u. a. 2007). Inzwischen gewinnen die mächtige Internet-Konzerne wie Apple, Google, Amazon und Facebook über ihre Märkte und Interessen hinaus weiteren Einfluss und setzen aufgrund ihrer wirtschaftlichen und technologischen Monopolstellung ihre in der Wirkung nicht nur technischen Standards für die eigenen Aktionsbereiche durch, zumindest solange ihnen keine ernsthaften Wettbewerber erwachsen. Kurzum, schon von der Vielzahl der relevanten Akteure her stellt die Internet Governance einen hybriden Policy-Mix dar, kompliziert noch durch den Faktor, dass die Reichweite ihrer jeweiligen Gestaltungsmacht im Zeitablauf deutlichen Veränderungen unterworfen ist.

\subsubsection{Internet Association for Assigned Numbers and Names (ICANN)}

Die Konstruktion und Aufgabenstellung der Internet Association for Assigned Numbers and Names (ICANN) lässt sich nur vor dem Hintergrund ihrer Vorgeschichte verstehen. Seit den späten 1970er Jahren hat sich die Koordination und das Management der Kernressourcen des Internet als ein von unten nach oben gesteuerter Prozess entwickelt, getragen von technischen Experten und den Nutzern, ohne Einmischung von staatlichen Stellen, ganz im Gegensatz zum Fernmelde- und Rundfunkwesen. Die notwendigen Standards zum Betreiben des Internets wurden von einer nicht-staatlichen Organisation, der Internet Engineering Task Force (IETF), einer informellen Gruppe von Technikern, entwickelt und angewandt. Auch das System der Namensräume (Domain Names) ist von der Basis aus entstanden, durch die Initiative des amerikanischen Informatikers von Jan Postel (1943-1998), der es von seinem Büro in Kalifornien aus entwickelte.

Bis Ende der 1990er Jahre wurde das Internet von den meisten Regierungen mehr oder weniger ignoriert. Als die Zahl der Halter von domain names stark anwuchs, begann sich die amerikanische Regierung für die Institutionalisierung dieses Systems zu interessieren; ein Vertrag zwischen Jan Postels Institut und dem US-Handelsministerium schuf die Internet Assigned Numbers Authority (IANA) zur Koordination der Internetressourcen, also zum Management der top level domains und der Zuweisung von IP-Adressen an die regionalen Internetregistrare. 
Mit der weiteren Zunahme von Haltern für Domain Names, von daraus wachsenden Konflikten um deren Zuteilung (plus steigenden Einnahmen aus der Registrierung) wuchs der Wunsch nach stärkerer Institutionalisierung. Jan Postels Bestreben, das System der Internet Society zu unterstellen, einem Netzwerk von Internettechnikern, scheiterte am Widerstand der amerikanischen Regierung (später auch der EU) und Teilen der Privatwirtschaft. Die US-Regierung plädierte 1997 stattdessen für eine vollständige Privatisierung des Domain Name-Systems auf der Grundlage kalifornischen Rechts, die EU revoltierte gegen die damit verbundene amerikanische Dominanz im System. Jan Postel wollte das gemeinsame Management der technischen Internet-Organisationen, der ITU und der WIPO unterstellen. Die USA und ihre Privatwirtschaft hatten aber starke Reserven gegen eine bedeutende Rolle der ITU, in der jedes Land gleichermaßen nur eine Stimme hat. Heraus kam als ausgehandelter Kompromiss eine neue, nicht-staatliche und nicht profitorientierte Organisation zum Management der Internet-Ressourcen unter kalifornischem Recht, die ICANN.

Grundgedanke ihrer Schaffung war das gemeinsame Management der Ressourcen durch Anbieter und Nutzer der Dienste, bei lediglich beratender Rolle nationaler Regierungen. Daher sollten 19 Mitglieder des Direktoriums von den Diensteanbietern gestellt werden und neun die breite Öffentlichkeit vertreten. Nationale Regierungen erhielten keinen Sitz, bestückten aber das Governmental Advisory Committee zur Beratung von ICANN (Kleinwächter 2001, 2004; Hofmann 2005). Die US-Regierung blieb - geplant zunächst für eine zweijährige Übergangsperiode, tatsächlich aber bis heute - das oberste Aufsichtsorgan für ICANN, der wichtigste Root Server steht in den USA. Die Direktoren der ICANN wurden im Jahre 2000 in der ersten und einzigen Wahl im Internet nach regionalem Schlüssel gewählt, wobei ein heftiger (nationaler) Konkurrenzkampf um die Besetzung stattfand. Als Folge wurde später die Beteiligung der Nutzer auf eine nicht stimmberechtigte Verbindungsfunktion reduziert.

ICANN war darüber hinaus mit einigen Geburtsfehlern behaftet, welche die späteren Kontroversen erklären: Die sich intensivierenden Konflikte um die Zuteilung von Domain Names vermochte die Organisation kaum zu dämpfen. Die regionalen Namensregistraturen weigerten sich teilweise, sich der vertraglichen Kontrolle von ICANN zu unterwerfen, die Finanzierung der Organisation blieb prekär. Sie blieb US-dominiert, das System der Domain Names war nicht multilingual, nationale Regierungen hatten darauf keinen oder nur geringen Einfluss. Das Gewicht der privaten Unternehmen in der Organisation war beherrschend, das der europäischen Staaten moderat, dasjenige der Entwicklungsländer sehr gering. Letztere argumentierten, technische Fragen (vor allem die Vergabe von Domain Names) seien durchaus politischer und nicht nur technischer Natur, sie wollten den amerikanischen Einfluss zurückdrängen - zehn der dreizehn Root Server blieben in den USA - und 
verlangten Mehrsprachigkeit und Unterstellung der ICANN unter die Vereinten Nationen oder die ITU (Cukier 2005; Pickard 2007).

Die Führungen dieser beiden Organisationen haben die Marginalisierung ihrer Organisation nie verwunden und agitierten vor allem vor und auf den Weltinformationsgipfeln dagegen. Sie und die WIPO (World Intellectual Property Organization), die beauftragt wurde, ein Schlichtungswesen für die Vergabe von Domain Names zu entwickeln, waren im Government Advisory Committee (GAC) vertreten (Mueller u. a. 2007). Dieses Gremium stellte durchaus einen Kanal dar, durch den nationale Regierungen ihren Einfluss geltend machen konnten. Doch es litt daran, dass anfangs längst nicht alle Regierungen als GAC-Mitglieder akkreditiert waren. Noch weniger nahmen an seinen Sitzungen regelmäßig teil. Dabei ist die Macht dieses Gremiums deutlich gewachsen; es hat nur noch de iure lediglich beratende Funktion, de facto aber die Vetomacht über die Entscheidungen von ICANN (Kleinwächter 2004).

Die Kontroverse über den Einfluss in der ICANN, über ihre Struktur und Arbeitsweise setzte sich bei den zwei Weltinformationsgipfeln und im Internet Advisory Forum fort. Weil man sich dort nicht auf eine Ersatzinstitution oder eine grundlegende Reform von ICANN einigen konnte, wurde die Debatte im Internet Governance Forum (IGF) fortgeführt, das auf dem 2. Gipfel ins Leben gerufen worden war. Mittlerweile sind selbst der amerikanischen Regierung Zweifel an der ursprünglich basisdemokratischen, regierungsfernen „Multi-Stakeholder“ getragenen Governance in der ICANN gekommen. Sie hat ihre Kontrolle über die Organisation zum September 2009 faktisch aufgegeben und spricht sich für eine stärkere Kontrolle durch die Regierungen aus (Mac Sitigh 2010; United Nations 2011). Dieser Meinung ist die Kommission der EU schon lange. Sie sprach sich sogar für ein Vetorecht der Regierungen über die Vermehrung von Top Level Domains (TLD) aus und verlangte, dass Ratschläge des Government Advisory Committee ernster genommen werden. Ferner kritisierte sie, dass die Finanzmittel und das Personal von ICANN ein zu schnelles Wachstum aufweisen und das Direktorium nicht wirklich unabhängig sei (European Commission 2011a). Hintergrund dieser scharfen Attacken ist - das wird auch implizit zugegeben - die wachsende wirtschaftliche und politische Bedeutung des Netzes.

\subsubsection{Die Weltinformationsgipfel}

Die Initiative für den Weltinformationsgipfel im Dezember 2003 in Genf kam im Wesentlichen von der ITU. Diese Organisation hatte relativ spät das enorme Potential des Internets, den globalen Wandel voranzutreiben und so genannte 
Informationsgesellschaften zu installieren, entdeckt. Eher hatte sie zunächst einem gewissen technologischen Fetischismus hinsichtlich des Netzes gefrönt. Diese Einschätzung sollte sich mit einer gemeinsam mit der UNESCO verfassten Studie (1995) ändern, die das Recht zur Kommunikation zum Inhalt hatte, den mangelnden Zugang zum Netz in vielen Ländern und das Fehlen einer universalen Infrastruktur für die Telekommunikation beklagte. Schuld daran wurde historischen Umständen (sprich: postkolonialen Strukturen) und der monopolistisch verfassten Medienindustrie gegeben. Diese Studie setzte damit einen Kontrapunkt zum Projekt der Informationsgesellschaft von Seiten der Industriestaaten, wonach Privatunternehmen die Initiative für Innovation und Investitionen (primär für kommerzielle Zwecke) bekommen sollten, wobei nationale Regierungen quasi als Juniorpartner fungieren, aber zivilgesellschaftliche Gruppierungen ausgeschlossen bleiben sollten (Raboy 2004). Gegen dieses Konzept liefen letztere Sturm, sie forderten Freiheit der Meinungsäußerung als Menschenrecht und kulturelle Pluralität im Netz ein.

Die ITU, welche den Prozess zum Weltinformationsgipfel 1998 startete, reagierte nur zögerlich auf diese Forderungen und erwähnte die Zivilgesellschaft als Beteiligte zunächst nicht. Das änderte sich erst, als sich eine zunehmend breitere NGO-Plattform bildete, die 2001 ihre Beteiligung an der Planung und der Durchführung des Gipfels forderte. Nach der Verbreiterung der Kampagne erhielt diese Rückendeckung durch die Führung der ITU und die Organisatoren des Weltinformationsgipfels, obwohl sich zahlreiche Regierungen gegen die Teilnahme der Zivilgesellschaft ausgesprochen hatten. Die UN-Generalversammlung segnete ihre Beteiligung jedoch ab, so dass ihre Vertreter dann aktiv an den Vorbereitungssitzungen des Gipfels teilnehmen konnten. Die ITU definierte eine breite Gipfelagenda mit dem Ziel, eine gemeinsame globale Vision der Informationsgesellschaft zu definieren. Außerdem thematisierte sie die digitalen Unterschiede und Spaltungen (digital divide) und formulierte das Ziel, das Internet in den Dienst aller sozialen Gruppen zu stellen. Diese Intentionen einschließlich einer pauschalen Forderung, mit dem Internet die Milleniumsziele der Vereinten Nationen zu erreichen, bestritten die ursprüngliche Agenda des Gipfels.

An ihm beteiligten sich über 10.000 Delegierte, wobei die Zivilgesellschaft und Vertreter der Wirtschaft nur Beobachterstatus hatten. Letztere waren durch strategische Partnerschaften mit einzelnen Staaten faktisch besser vertreten. Obwohl eine breite Palette von Themen diskutiert werden sollte, entwickelte sich die Frage der Internet Governance zu ihrer wichtigsten und am kontroversesten verhandelten, andere Themen - wie die Finanzierung eines Solidaritätsfonds zugunsten armer Staaten - traten deshalb in den Hintergrund. Die Entwicklungsländer und die meisten Organisationen der Zivilgesellschaft stellten vor allem die Autorität und 
Legitimität von ICANN in Frage, bemängelten die Dominanz der Industriestaaten und der Unternehmen in dieser Organisation sowie die willkürliche Vergabe von Domain Names und strebten eine Verlagerung der Kompetenzen von ICANN zur ITU an. Die versammelte Zivilgesellschaft sorgte sich um mangelnde kulturelle Diversität, die fehlende Berücksichtigung indigener Völker und von Geschlechtergleichheit und kritisierte den technokratischen und wirtschaftsfreundlichen Ansatz der Konferenzagenda. Die Handelskammern und Unternehmen aus Industriestaaten sowie die Vertretung der USA und der EU zeigten sich mit der bisheriger Struktur der Internet Governance dagegen durchaus zufrieden und gaben nur zu bedenken, dass die Beteiligung einer UN-Organisation an dieser Governance das Internet bürokratisieren und Innovation hemmen würde (Kleinwächter 2004; Pickard 2007).

Wie die meisten internationalen Konferenzen segnete der Gipfel einen ambitionierten Aktionsplan ab - mit dem Ziel, eine inklusive und offene Informationsgesellschaft zu schaffen, die demokratisch regiert werden, kulturelle und Geschlechtergleichheit fördern soll, ohne konkret anzugeben, wie diese Ziele erreicht werden könnten. Fragen ungleicher Machtverteilung in der Weltinformationsgesellschaft, Konzentrationstendenzen bei privaten Medienunternehmen und das Schrumpfen des Raums öffentlicher Medien wurden weitgehend tabuisiert. Internationale Abkommen, wie das 1994 vereinbarte Übereinkommen über handelsbezogene Aspekte der Rechte am geistigen Eigentum (englisch: Trade Related Intellectual Property Rights, TRIPSs) im Rahmen der Welthandelsorganisation (World Trade Organization, WTO), das bestimmte Anforderungen an nationale Verfahren zum Schutz geistiger Eigentumsrechte stellt, damit sie nicht durch den grenzüberschreitenden Handel unterlaufen werden können (Shadlen 2007), oder das Recht auf geistiges Eigentum im Rahmen der WIPO-Konventionen, welche den nationalen, medienpolitischen Spielraum beschränken, wurden nicht weiter problematisiert. Hingegen wurden Informationstechnologien als wirkungsvolle Waffe gegen Unwissenheit stilisiert (Hamelink 2004; O'Siochru 2004). Aus Frustration über die wirtschaftsnahe Verengung der Agenda zog sich die Zivilgesellschaft zuletzt aus dem Gipfelprozess zurück und verfasste eine eigene Schlussdeklaration.

Die hauptsächlich strittigen Themen - also die Einrichtung eines digitalen Solidaritätsfonds zur Überwindung der digitalen Spaltung und die engere Frage der Zukunft von ICANN als Kernorganisation der Internet-Governance - wurden auf den nachfolgenden Gipfel (2005) in Tunis verschoben. Zur Klärung dieser Frage sollte der UN-Generalsekretär eine Arbeitsgruppe einrichten. Ihr gehörten Vertreter der Regierungen, der Zivilgesellschaft und der Privatwirtschaft an, und sie sollte Vorschläge für die Nachfolgekonferenz erarbeiten. Kritische 
Beobachter monierten indes, dass sich gemäßigte Vertreter der Zivilgesellschaft in die Strategie der Konferenz trotz des eigenen Schlusskommuniques hätten einbinden lassen, dass die ITU gegenüber den Interessen der mächtigsten Staaten kapituliert und ihren marktliberalen Ansatz der Informationsgesellschaft akzeptiert habe (McLaughlin und Pickard 2005; Pickard 2007).

Die eingesetzte Arbeitsgruppe zur Internet Governance (Working Group on Internet Governance, WGIG) sollte eine Arbeitsdefinition von Internet Governance liefern, mit dieser wesentliche politische Fragestellungen herausarbeiten und für ihre Umsetzung eine praktikable Aufgabenverteilung zwischen Regierungen, internationalen Organisationen, der Wirtschaft und der Zivilgesellschaft vorschlagen. Doch der Bericht dieser Gruppe von ca. 40 Experten blieb relativ vage. Governance wurde sehr breit definiert. Als wesentliche Fragestellungen wurden relativ allgemeine und prinzipiell lobenswerte Ziele aufgelistet (Zugang für alle zum Netz, stabiles Funktionieren des Internet, gerechte Verteilung der Ressourcen etc.). Ferner sollte Kontrolle über die Internet Governance durch ein ,Multi-Stakeholder'-Forum erfolgen, dessen Arbeitsweise unklar gelassen wurde, in dem aber Entwicklungsländer stärker vertreten sein sollten. Die Regierungen sollten politische Fragestellungen bestimmen, die täglichen Operationen jedoch der Privatwirtschaft und der Zivilgesellschaft überlassen bleiben - so als ob man beides voneinander trennen könne (Mueller u. a. 2007). Bei der Kontrolle durch die Regierungen wurden vier Modelle aufgeführt, ohne sich klar für eines auszusprechen. Mithin wurde auch in dieser Gruppe kein tragfähiger Konsens erreicht.

Die USA ließen alsbald verlauten, sie würden die Kontrolle über ICANN nicht aufgeben, was sehr zum Unwillen auch der Europäischen Union erfolgte. Auf dem Nachfolgegipfel in Tunis (im November 2005), der unter noch stärkerer Beteiligung stattfand, knickte die EU nach anfänglich scharfer Attacke (ebenso wie andere Teilnehmer) gegen die USA wieder ein; es blieb beim Status quo. Die strittige Frage wurde an ein neu geschaffenes Gremium unter der Regie der Vereinten Nationen, an das Internet Governance Forum (IGF), delegiert, das einen evolutionären Prozess zu einer stärker multilateral organisierten InternetGovernance einleiten sollte (McLaughlin und Pickard 2005; Pickard 2007). Kurzum, vier Jahre dauernde Verhandlungen brachten wenig Greifbares ein.

\subsubsection{Das Internet Governance Forum (IGF)}

Das IGF war im Wesentlichen als Diskussionsforum für alle Aspekte der Internet Governance gedacht. Es sollte Vorschläge entwickeln, wie die Verbreitung des Netzes 
in Entwicklungsländern zu fördern ist und dort Kapazitäten ausgebaut werden können. Außerdem sollte es Lösungen entwickeln, um den Missbrauch des Netzes zu unterbinden. Die zunächst 40, später 50 Mitglieder seiner Multistakeholder Advisory Group werden von Regierungen, der Privatwirtschaft, der Zivilgesellschaft gestellt und um technische Experten bereichert. Das IGF wurde erstmals im Spätherbst 2006 durch den Generalsekretär der Vereinten Nationen einberufen und erhielt zunächst ein Mandat für fünf Jahre, das mittlerweile verlängert wurde (2011 bis 2015). Bei der ersten Sitzung des IGF in Athen wurde eine ganze Reihe ,dynamischer Koalitionen" gegründet; das sind informelle Diskussionsgruppen zu bestimmten Themen der Internet Governance, etwa zum Datenschutz, zur Mehrsprachigkeit im Netz, zur Meinungsfreiheit, zur Anbindung abgelegener Regionen und zum Jugendschutz. Beim Treffen in Rio de Janeiro (2007) ging es wieder um die Zukunft von ICANN und den Einfluss von Regierungen auf die Zukunft des Netzes, um Mehrsprachigkeit, offene Netz-Standards und die Sicherheit von Internet-Transaktionen. Zugang für alle zum Internet war das Thema des dritten Treffens in Hyderabad (2008). Einen ähnlichen Fokus hatte das vierte Treffen in Sharm El Sheikh (2009), und bei den beiden letzten Konferenzen wurde erneut die eigentliche Internet Governance thematisiert. Diese Versammlungen fanden unter starker Besetzung (über 1000 Teilnehmer), statt. Jeweils ca. 40 Prozent der Teilnehmer entfielen auf Regierungsvertreter. Wie nicht anders zu erwarten, blieben die Entwicklungsländer wegen fehlender Reisemittel und Expertise unterrepräsentiert.

Der Bericht des UN-Generalsekretärs kritisiert (recht milde) die mangelnde Effizienz der ausufernden Debatten, den Mangel an konkreten Empfehlungen für das Regierungshandeln und die fehlende Berücksichtigung des eigentlichen Kernthemas (dem Management der Internet-Ressourcen); er lobt allerdings rituell den multilateralen, demokratischen und transparenten Stil dieses Unterfangens und empfiehlt seine Fortsetzung (United Nations 2010). Unabhängige Beobachter waren da weitaus kritischer; sie beklagten die Fortsetzung der alten Kontroversen (nämlich: stärkere staatliche Kontrolle versus Selbstorganisation) im neuen Gewande, den Mangel an Dialog zwischen den Opponenten und die Verabschiedung von Papieren voller Gemeinplätze (Maciel 2011). Kurzum, Aufwand und Ertrag standen und stehen in einem starken Missverhältnis.

\subsubsection{Die UNESCO}

Die Verfassung der UNESCO (United Nations for Educational, Scientific and Cultural Organization) wurde im November 1945 von Vertretern von 37 Staaten unterzeichnet und trat 1946 in Kraft. Ihre Gründung geht auf eine Initiative der Erziehungsminister der Alliierten zurück. Ihre nachkriegsbedingten Leitbilder 
waren der Abbau von Feindbildern, die Förderung der Zusammenarbeit der Völker bei Bildung, Wissenschaft und Kultur und die Erziehung zum Frieden. Rechtlich hat die UNESCO den Status einer Sonderorganisation der Vereinten Nationen. Die Mitgliedschaft in den Vereinten Nationen berechtigt auch zu jener in der UNESCO.

Heute sind fast alle Staaten Mitglieder der Organisation (insgesamt 195). Zwischenzeitlich traten allerdings Großbritannien und die USA wegen angeblichem Missmanagement und Politisierung der Organisation aus. Auch in der UNECSO gilt das Prinzip „ein Staat = eine Stimme“. Beschlüsse werden allerdings seit Jahrzehnten vorwiegend einvernehmlich gefasst. Die UNESCO ist im Verlauf ihrer Geschichte zunehmend zu einem Instrument der Mitgliedsstaaten geworden, die Mitglieder des Exekutivrates sind heute ausschließlich Politiker (und nicht, wie anfangs vorgesehen, auch Vertreter des Geisteslebens). Das Sekretariat mit etwas über 2000 Mitarbeitern und mit Sitz in Paris wird von einem Generaldirektor geleitet und ist in etliche, mitunter schwer zu koordinierende Abteilungen gegliedert. Eine Besonderheit der UNESCO ist die Einrichtung nationaler UNESCO-Kommissionen, die in einer Brückenfunktion die Mitgliedsstaaten beraten und als Verbindungsstellen zu lokalen Nichtregierungsorganisationen fungieren. Der Haushalt der UNESCO ist vergleichsweise bescheiden (2012/13: 653 Mio. \$ für zwei Jahre), er entspricht gerade einmal jenem einer mittleren deutschen Hochschule. Neben den Mitteln aus dem normalen Haushalt stehen der UNESCO auch noch außerordentliche Mittel zur Verfügung, die primär für die Durchführung von Entwicklungsprojekten bestimmt sind.

Gemäß ihrer Verfassung hat die UNESCO folgende Aufgaben: (a) die gegenseitige Kenntnis und das Verständnis der Völker durch Nutzung der Medien zu fördern und Abkommen zu empfehlen, die den freien Austausch durch Wort und Bild erleichtern, (b) der Volksbildung und der Verbreitung der Kultur neue Impulse zu geben, (c) das kulturelle Erbe der Welt zu bewahren, zu vertiefen und zu verbreiten. Praktische Arbeitsschwerpunkte der Organisation sind heute die universale Alphabetisierung und Grundbildung, neuerdings auch die Berufsbildung und das lebenslange Lernen, der Aufbau wissenschaftlicher Forschungs- und Ausbildungsinstitutionen in Entwicklungsländern, die Wahrung des Weltkulturerbes (etwa durch die Erstellung einschlägiger Listen von Denkmälern), das Einsetzen für Presse- und Informationsfreiheit und die Friedenserziehung. Gemäß ihrer bescheidenen Finanzausstattung kann die UNESCO jedoch kaum mehr als ein Forum zum Austausch von Meinungen und Erfahrungen in ihren Aufgabenfeldern bieten sowie einige Pilotprojekte finanzieren.

Von Beginn an gab es Konflikte darüber, welchen Aufgaben sich die UNESCO widmen und welcher weltanschaulichen Richtung sie verpflichtet sein sollte. Diese Konflikte verschärften sich mit dem zunehmenden Gewicht der Entwicklungsländer in der Organisation, die - zusammen mit den (damals) sozialistischen Staaten - schon in den 1970er Jahren eine neue, nicht von 
westlichen Medienkonzernen dominierte Weltinformationsordnung durchdrücken wollten. Dies war Hauptgrund für den zeitweiligen Austritt der USA und Großbritanniens. Heute ist es um diese Debatten recht still geworden.

Die UNESCO kreist in ihren Aktivitäten und Publikationen schon seit Jahren um den Begriff und den Inhalt einer "globalen Wissensgesellschaft", die mitunter auch unter dem Stichwort "Digitales Zeitalter" rubriziert wird. Digitale Technologien und neue Medien übernehmen nach Meinung der Organisation zentrale Funktionen in unserem Leben. Die Organisation will dabei helfen, möglichst viele Menschen in relativ kurzer Zeit zu digitalen Alphabeten auszubilden und dafür zu sorgen, dass sie lebenslang ihr diesbezügliches Wissen aufstocken (Varis o.J.). Um menschlich und lebenswert zu bleiben, müssten so genannte Wissensgesellschaften für die möglichst allgemeine, gründliche und egalitäre Verbreitung von wahrhaftigem und relevantem Wissen sorgen. Daher wird einesteils die erhebliche digitale Spaltung zwischen Nord und Süd, aber auch innerhalb der Gesellschaften beklagt; andererseits wird gefordert, dass die Informations- und Kommunikationstechnologien dazu genutzt werden, die menschliche Entwicklung auf der Basis garantierter Menschenrechte zu fördern. Gemeint ist damit einerseits die Sicherung der Meinungsfreiheit, denn ohne diese mache Zugang zur Information alleine keinen Sinn. Andererseits spricht sich die UNESCO gegen die exzessive Kommerzialisierung von Wissen durch die neuen, in Produktionsprozesse integrierte Datenbasen und Suchmaschinen aus (UNESCO 2005).

Außerdem begrüßt die UNESCO, dass durch die Entwicklung des Internets der freie Informationsfluss enorm gesteigert wird. Diese Entwicklung berge erhebliches Potential für globalen kulturellen Dialog, internationale Verständigung und politische Partizipation, vorausgesetzt, die digitale Spaltung werde verringert. Daher müsse Internet Governance eine gerechte Verteilung der Ressourcen sichern sowie den Zugang aller zum Netz und sein stabiles Funktionieren auf möglichst multisprachlicher und -kultureller Basis („UNESCO's activities in the area of Internet Governance“) ermöglichen. An dieser Governance müssten alle Akteure beteiligt werden.

\subsubsection{Die World Intellectual Property Organization (WIPO)}

Die Weltorganisation für geistiges Eigentum wurde 1967 in Stockholm gegründet und 1974 zu einer der Sonderorganisationen der UN mit Sitz in Genf erhoben. Zweck der WIPO ist es, den Schutz des geistigen Eigentums durch globale Kooperation zu fördern. Geistiges Eigentum wird dabei breit verstanden und umfasst alle Rechte, die sich aus gewerblichen, wissenschaftlichen, literarischen 
und künstlerischen Tätigkeiten ergeben; außerdem ist der Schutz gegen unlauteren Wettbewerb einbeschlossen. Das Eigentum bezieht sich auf den Schutz von Erfindungen, Warenzeichen und gewerblichen Mustern und Modellen, das Urheberrecht auf den Schutz von literarischen, musikalischen Werken und solchen der bildenden Kunst, der Fotografie, des Films und der Audiovision. Die WIPO verwaltet Verbände bzw. Verträge zum Schutz einzelner Bereiche des geistigen Eigentums, nämlich den Pariser Verband zum Schutz gewerblichen Eigentums und den Berner Verband zum Schutz von Werken der Literatur und der Kunst.

Die Aufgaben der WIPO sind der Abschluss neuer internationaler Vereinbarungen und die Modernisierung der nationalen Gesetzgebung zum Schutz des geistigen Eigentums, technische Hilfen für Entwicklungsländer, der möglichst globale Schutz von Erfindungen, Warenzeichen und gewerblichen Mustern sowie die Zusammenarbeit der Mitgliedsländer in diesen Bereichen. Die Mitgliedschaft zur WIPO steht jedem Staat offen, der einem der beiden Verbände angehört (zur Zeit 185 Mitgliedsstaaten) oder - gleichsam als Teilhaber zweiter Klasse - wer Mitglied der Vereinten Nationen ist. Das wichtigste Organ der WIPO ist das Internationale Büro für geistiges Eigentum; es unterhält fünf internationale Registrierungsdienste (Patente, Marken, gewerbliche Muster, Ursprungsbezeichnungen und audiovisuelle Werke). Die WIPO finanziert sich durch gestaffelte Mitgliedsbeiträge und Gebühren für die Registrierung von Anmeldungen zum Schutz des geistigen Eigentums.

Beim Schutz gewerblichen Eigentums wurde kein einheitliches Recht geschaffen, sondern die so genannte Inländerbehandlung umgesetzt, $\mathrm{d}$. h. alle Gewerbetreibende, die einem Verbandsstaat angehören, genießen innerhalb eines Vertragsstaates die gleichen Rechte. Ähnliches gilt für den Markenschutz. Bei Patenten ist eine internationale Anmeldung möglich gemacht worden, bei der alle Staaten benannt werden können, in denen ein Patent angestrebt wird. Das Urheberrecht (für Literatur, Musik, Kunst, Filme etc.) schützt den Schöpfer ohne Zahlungen und Registrierung, in der Regel für 50 Jahre Dauer. Innerhalb der Organisation gab es langjährige Konflikte zwischen den Industrieländern, die auf verstärkten Schutz des geistigen Eigentums drängten, und den Entwicklungsländern, die zum Zweck ihrer wirtschaftlichen Entwicklung einen verstärkten Transfer dieses Eigentums und damit eine Aufweichung des Patentschutzes forderten. Die Industrieländer brachten darauf das Thema des geistigen Eigentums in die Uruguay-Runde des Allgemeinen Zoll- und Handelsabkommens (General Agreement on Tariffs and Trade, GATT), also der mit schwächeren Vollmachten ausgestatteten Vorgängerorganisation der WTO, ein und erreichten dort eine Übereinkunft über die handelsbezogenen Rechte an geistigem Eigentum (= TRIPS-Abkommen). Es firmiert heute unter dem Dach der Welthandelsorganisation (WTO) und regelt die Rechte der Eigentümer im internationalen Handelsverkehr umfassend. 
Gemäß ihrer allgemeinen Aufgabenstellung hat sich die WIPO mit Fragen zum Internet befasst, die sich für den Schutz des geistigen Eigentums ergeben. Einschlägig sind dafür

(a) der WIPO Copyright-Vertrag vom Dezember 1996, der die Berner Übereinkunft aufrecht hält, sie auf die elektronische Vervielfältigung ausdehnt und dem Internet-Zeitalter anpasst, also z. B. auch Computerprogramme und Datenbanken unter Copyright-Schutz stellt.

(b) Der WIPO Performances and Phonograms Treaty (ebenfalls von Dezember 2006) wendet den Schutz des geistigen Eigentums von Musik-Künstlern, Schallplattenproduzenten und Rundfunksendern (Vertrag von Rom, 1961) auf die Verbreitung ihrer Werke über das Internet an. Beide Verträge wurden von etwas mehr als 30 Staaten unterzeichnet und sind 2002 in Kraft getreten.

(c) Ein dritter internetbezogener Vertrag (Bejing Treaty on Audiovisual Performances) soll - wie der Name schon sagt - das geistige Eigentumsrecht audiovisueller Produktionen im Internet schützen. Er wurde im Juni 2012 in Peking vereinbart, ist aber noch nicht in Kraft getreten.

(d) Schon 1998 verfasste die WIPO - ausgehend von einem Antrag der amerikanischen Regierung - Empfehlungen zum Schutz geistigen Eigentums, die mit den Domain Names verbunden sind. Sie wurden an ICANN und die Mitgliedsstaaten der WIPO zur Beratung überstellt. Im Kern ging es darum, die Überschneidung von Markennamen und Domain Names zu vermeiden bzw. zu regeln. Es folgte im Jahr 2002 auf Wunsch etlicher Mitglieder eine zweite Runde, die sich vor allem der missbräuchlichen Verwendung von persönlichen Namen und jenen internationaler Organisationen, von geographischen Bezeichnungen, Handelsmarken und pharmazeutischen Substanzen widmete. Auch der zweite Bericht wurde auf Wunsch der Mitgliedsstaaten ICANN überstellt, von dieser Organisation aber nur teilweise umgesetzt.

(e) Seit 1999 bietet das Arbitration and Mediation Centre der WIPO Streitschlichtung für Konflikte um Generic Top-Level Domains (gTLDs wie .org oder .com) an. Die Organisation arbeitet mit ICANN zusammen, so dass vor allem Markennamen bei der Einführung neuer gLTDs geschützt werden. Streitfälle werden von einer unabhängigen Arbeitsgruppe entschieden.

\subsubsection{Die International Telecommunication Union (ITU)}

Die Internationale Fernmeldeunion hat eine lange Vorgeschichte, ihre Vorgängerinstitutionen wurden 1934 zusammengefasst. Nach dem Zweiten Weltkrieg 
(1947) wurde die ITU umstrukturiert und $1949 \mathrm{zu}$ einer Sonderorganisation der Vereinten Nationen gemacht (mit Sitz in Genf). Die Mitgliedschaft steht allen Mitgliedsländern der Vereinten Nationen sowie allen übrigen Ländern offen, für deren Beitritt sich zwei Drittel der Mitglieder erklären. Der ITU gehören zur Zeit 193 Länder an. Eine Besonderheit der ITU als einzige UN-Sonderorganisation ist, dass sie eine spezielle Partnerschaft zwischen Vertretern der Regierungen und der einschlägigen Wirtschaft aufweist. Alle namhaften Telekommunikationsfirmen (zur Zeit über 700) sind sogenannte "Sector Members" der Organisation. Der ITU obliegt die internationale Ordnung des Fernmeldewesens. Sie soll die internationale Zusammenarbeit der Fernmeldedienste erhalten und ausbauen, die technische Entwicklung und die sektorspezifische Zusammenarbeit der Staaten fördern. Insbesondere ist sie für die Aufteilung des Frequenzspektrums für die verschiedenen Funkdienste, die Schaffung weltweiter Standards im Fernmeldewesen und für den Ausbau von Telekommunikationseinrichtungen vor allem in Entwicklungsländern verantwortlich. Die Finanzierung der ITU erfolgt hauptsächlich durch Beiträge der Mitgliedsländer, ferner durch solche anerkannter privater Betriebsunternehmen. Die Mitglieder wählen ihre Beitragsklasse nach freiem Ermessen.

Wie kaum eine andere Sonderorganisation der Vereinten Nationen musste sich die ITU dauernd mit neuen technischen Entwicklungen in ihrem Kompetenzbereich befassen (zuletzt mit dem Internet). Sie hatte relativen Erfolg bei der technischen Absicherung der globalen Weiterentwicklung der Kommunikationssysteme, der Übertragung von Hörfunk- und Fernsehprogrammen sowie des weltweiten Datentransfers. Die damit verbundenen wirtschaftlichen, sozialen und kulturellen Probleme wurden vorwiegend in anderen internationalen Organisationen debattiert, so dass der ITU größere interne Konflikte erspart blieben.

Die ITU ist als explizit technische Organisation in alle Phasen der InternetEntwicklung involviert, sie ist vornehmlich bei der Entwicklung digitaler Zertifizierung, der Standardisierung der Tarifmodelle, der Übertragungsmodi, der Sicherung von Qualität der Dienste sowie dem Telefondienst über Internet beteiligt. Die ITU setzt sich seit längerem für die weitere Liberalisierung der Telekommunikationsdienste ein. Für das Internet vertritt sie, dass die nationalen Domain Names Ressourcen des jeweiligen Landes darstellen und von dessen Behörden letztinstanzlich verwaltet werden sollen. Sie ist für eine gerechtere Zuteilung künftiger IP-Adressen nach Ländern und versteht sich auch als Anwalt der ärmeren Länder bei der Unterstützung internetbezogener Projekte, von Ausbildungs- und Beratungsmaßnahmen.

Die Rolle von ICANN beurteilt die ITU im Vergleich $\mathrm{zu}$ anderen UNSonderorganisationen moderat: Sie betont den Willen zur weiteren Zusammenarbeit mit ICANN, lobt die Ansätze der amerikanischen Regierung zur Internationalisierung 
dieser Organisation, weist aber gleichzeitig auf das Legitimitätsdefizit von ICANN aufgrund der regional unausgewogenen Einflussverteilung in seinem Direktorium hin. Sie plädiert auch dafür, dass Entscheidungen über knappe Ressourcen (also auch Domain Names) und die Sicherheit des Internet von Regierungen getroffen werden sollten, und bietet sich dabei selbst als intergouvernementale Schaltstelle an (ITU and Internet Governance 2004).

Operativ war die ITU in den letzten Jahren vor allem auf dem Gebiet der "Cybersecurity" aktiv, also insbesondere bei der Abwehr von Cyberattacken, Kriminalität und terroristischen Aktivitäten im Netz durch globale Kooperation. Die ITU wurde als einzige internationale Organisation mit dieser Aufgabe auf dem Weltinformationsgipfel betraut. Sie hat dazu fünf Arbeitsgruppen eingesetzt, die folgende Felder abdecken sollen:

(a) Rechtliche Maßnahmen, insbesondere die Harmonisierung der Gesetze gegen Cyberkriminalität. Hierzu hat die Organisation einen einschlägigen Werkzeugkasten entwickelt, der vor allem die Bemühungen von Entwicklungsländern unterstützen soll und Blaupausen für rechtliche Regelungen gegen Cybercrime liefert.

(b) Technische Maßnahmen, d.h. die Entwicklung gemeinsamer Sicherungsstandards für Internetdienste, besonders auch für den E-commerce. Hierzu wurde ein Kompendium vereinbarter ITU-Standards publiziert.

(c) Organisatorische Strukturen, gemeint ist die Koordination der Abwehr von Cyberattacken, beginnend auf der regionalen Ebene.

(d) Schaffung von Informationskompetenzen, d. h. die Bildung der Endnutzer in Bezug auf mögliche Gefahren bei der Internetnutzung.

(e) Internationale Kooperation bei der Bekämpfung von Cybercrime und beim Jugendschutz im Netz (ITU 2008).

\subsubsection{Die Organization for Economic Cooperation and Development (OECD)}

Die Organisation für Wirtschaftliche Zusammenarbeit und Entwicklung geht auf den amerikanischen Marshall-Plan zur wirtschaftlichen und politischen Stabilisierung Europas nach dem Zweiten Weltkrieg und den einsetzenden Ost-West-Konflikt zurück. Sie wurde 1948 in Paris gegründet. Ihre unmittelbaren Aufgaben waren der wirtschaftliche Wiederaufbau Europas mit Hilfe amerikanischer Unterstützung und die Liberalisierung des Handels- und Währungsverkehrs zwischen den Mitgliedsländern. Nachdem diese Ziele weitgehend erreicht wurden, wandte sich 
die OECD weiteren Aufgabenfeldern zu, etwa der Koordination der westlichen Entwicklungshilfe und der verstärkten Abstimmung der Wirtschaftspolitik der Mitgliedsländer. Seither sind die Ziele der OECD die Förderung der wirtschaftlichen Entwicklung der Mitgliedsländer, Hilfe bei der wirtschaftlichen Entwicklung der ärmeren Staaten und die Ausweitung des Welthandels. Entscheidungen der OECD bedürfen der Einstimmigkeit, Stimmenthaltungen blockieren diese aber nicht. Die Organisation arbeitet mit einer Vielzahl von Fachausschüssen, die nicht immer für alle Mitglieder offen sind; die wichtigste Rolle nimmt der wirtschaftspolitische Ausschuss ein. Mit der OECD verbunden ist eine Reihe bekannter und weitgehend autonomer Institutionen wie die Atomenergie-Agentur und die Internationale Energie-Agentur.

Mit der Gründung und Erweiterung der Europäischen Gemeinschaft ist die Bedeutung der OECD etwas zurückgegangen; sie hat dies durch die Aufnahme außereuropäischer Mitglieder (neben den assoziierten Gründungsmitgliedern USA und Kanada nun Japan, Südkorea und Mexiko) etwas ausgleichen können. Die OECD ist ein wichtiges Organ zur gemeinsamen Diskussion und Abstimmung der Wirtschaftspolitik westlich orientierter Staaten geblieben. Dies geschieht auch im Rahmen von Konsultationen und von "peer reviews“, bei denen sich die Mitglieder der Überprüfung durch die anderen aussetzen.

Die OECD zeigt eine ausgesprochen positive Einstellung zur Entwicklung der Internetökonomie; sie sieht diese als wichtige Kraft für die Förderung von Innovation, wirtschaftlicher Produktivität und Wachstum und möchte diese daher gefördert wissen. Sie spricht sich auch für die Beibehaltung des MultiStakeholder-Ansatzes bei der Internet Governance aus, also für das Management des Netzes durch Regierungen, den privaten Sektor, technische Experten und die Nutzer. Sie hat sich früh für die Förderung der digitalen Wirtschaft eingesetzt und fordert weiteres Engagement und Vertrauen für deren Aufbau. Ferner will sie den Zugang zum Internet für jeden ermöglichen, Innovation und Wettbewerb im System weiter unterstützen, den Schutz persönlicher Daten und des geistigen Eigentums sichern sowie den Ausbau der Internet-Infrastruktur vorantreiben helfen (OECD 2008c).

\subsubsection{Die Europäische Union (EU)}

Es würde an dieser Stelle zu weit führen, das ganze Panorama der Entstehung, Struktur, Institutionen, Rechtsgrundlagen und Tätigkeitsfelder der Europäischen Union auszubreiten. Wichtig ist jedoch im Kontext von Internet Governance, dass durch die EU-Verträge- zuletzt durch jenen von Lissabon (2009) - die Mitgliedsstaaten der 
Union Souveränitätsrechte übertragen haben, die EU also (begrenzt) supranationalen Charakter trägt und damit eigenen Rechtscharakter hat. Das heißt, die EU kann für die Mitgliedsstaaten politisch handeln, Gesetze erlassen und durchsetzen, allerdings nur im Rahmen der ihr zugestandenen Kompetenzen. Sie ist auch berechtigt, völkerrechtlich verbindliche Verträge abzuschließen und internationalen Organisationen beizutreten. Je nach Gesetzesmaterie entscheidet der Europäische Rat entweder einstimmig oder nach einfacher bzw. qualifizierter Mehrheit. Entscheidungen können einmal Verordnungen sein, die unionsweit gelten, verbindlich sind und über nationalem Recht stehen; ferner gibt es Richtlinien, die von den Mitgliedern in nationales Recht umgesetzt werden müssen; schließlich können Beschlüsse gefasst werden, die für die benannten Empfänger verbindlich sind und - zuletzt - sind unverbindliche Empfehlungen möglich. Der Europäische Rat und das Europäische Parlament besitzen gemeinsam die Haushaltsbefugnis.

$\mathrm{Zu}$ den gemeinschaftlich zu verfolgenden Politikbereichen in der EU gehören die Schaffung eines Binnenmarktes für Kapital, Arbeitskräfte, Waren und Dienstleistungen, die binnenmarktflankierenden Politiken (also die Kohäsions-, Sozial-, Außenwirtschafts- und Zugangspolitik), die binnenmarktangeregten Politiken (also die Umwelt-, Gesundheits- und Verbraucherpolitik) sowie die sektoralen Sonderpolitiken (für Kohle und Stahl, Landwirtschaft, Verkehr, Atomenergie). Andere Politikbereiche (Außen- und Sicherheitspolitik, justizielle und polizeiliche Zusammenarbeit) sind intergouvernemental angelegt und verbleiben also weitgehend in der Souveränität der Mitgliedsstaaten. Ausschließliche Zuständigkeit hat die EU nur bei der Durchführung der gemeinsamen Handelspolitik sowie bei der binnenmarktbezogenen Rechtsangleichung. In allen anderen Bereichen behalten die Mitgliedsstaaten das Recht auf eigenständiges Tätigwerden, das aber nicht in Widerspruch zum Gemeinschaftsrecht treten darf. Maßnahmen der Gemeinschaft dürfen nicht über das zur Erreichung der Ziele des EU-Vertrages erforderliche Maß hinausgehen; zudem muss der gemeinschaftliche Rechtsakt so weit wie möglich auf die nationalen Rechtsordnungen der Mitglieder Rücksicht nehmen (Müller-Graff 2002). Unschwer ist aber zu erkennen, dass vor allem die binnenmarktbezogenen Befugnisse der EU jene Aktivitäten einschließen, welche die hauptsächlichen Regelungen des InternetVerkehrs beinhalten.

Schon früh (1974) haben die Europäische Gemeinschaft und ihre Mitgliedsstaaten ein Aktionsprogramm für die Wissenschafts- und Technologiepolitik aufgelegt, das auch der Förderung von Informationstechnologien dienen sollte. Zweifel an der Wettbewerbsfähigkeit Europas vor allem gegenüber Japan und angebliche Rückstände bei fortschrittlichen Technologien verliehen diesen Bemühungen in den 1980er Jahren mächtigen Schub: Der sogenannte „Davignon-Bericht“ arbeitete die 
zentrale Rolle der Informationstechnik für die wirtschaftliche Zukunft Europas heraus und ermahnte zu gemeinschaftlichen, statt nur nationalen Anstrengungen. 1983 fiel die Entscheidung für ein vergleichsweise massives Forschungsrahmenprogramm mit dem Schwerpunkt auf den Informationstechnologien. Sein Ziel war es, mindestens technologische Parität mit den internationalen Wettbewerbern zu erreichen; das Programm wurde mehrfach aufgestockt, erweitert und fortgeschrieben (Sturm 2002). Mit der Einheitlichen Europäischen Akte (1986) wurde die Forschungs- und Technologiepolitik zur Gemeinschaftsaufgabe.

Die Europäische Union stellte sich auch relativ früh als ein wichtiger Akteur bei der internationalen Diskussion um das Internet-Management dar und beteiligte sich aktiv an den Weltinformationsgipfeln 2003 und 2005. Sie sprach sich von Beginn an für eine multilaterale (also nicht nur amerikanische) und stärkere staatliche Aufsicht über die Aktivitäten der ICANN, für eine Reform der Regeln, die zur Einführung neuer generic domains (z. B. .com, .org.) führen, für eine Trennung von „,registries" and ,registrars" activities“, also von regionalen Verzeichnissen und Registraren, sowie für einen Transfer des Root-ServerManagements vom US-Handelsministerium zu ICANN aus.

Außerdem legt die Europäische Kommission großen Wert auf den Erhalt einer offenen, neutralen und interoperablen, also über Ländergrenzen hinweg anschlussfähigen Internet-Architektur. Sie will, dass der Privatsektor weiter die Führung über die nötigen Investitionen in die Netze und die unternehmerische Initiative bei der Entwicklung von Innovationen und der Verbreitung des Internetanschlusses behält. Gleichzeitig stellt sie aber fest, dass das Internet zu einer kritischen Entwicklungsressource geworden ist, bei der jede Unterbrechung des Angebots gesellschaftlich katastrophale Folgen haben könnte. Die meisten Nutzer erwarten ein verlässliches Angebot und machen Regierungen für Defizite verantwortlich. Aus dieser Motivation wird gefolgert, dass nationale Regierungen (und die EU) größeren Einfluss auf relevante Entscheidungen zur Weiterentwicklung des Netzes nehmen und dass private Agenturen zum Management der Netze gesellschaftlich verantwortlich gemacht werden müssten. Davon ausgenommen sind tägliche Routineentscheidungen (European Commission 2009a).

Die Kommission der EU verfasste 2010 eine Digitale Agenda für Europa (Europäische Kommission 2010b), die das Ziel formuliert, in Europa einen digitalen Binnenmarkt zu schaffen, der auf einem schnellen Internet beruht und nachhaltigen wirtschaftlich-sozialen Nutzen schaffen soll. Die digitale Agenda versteht sich als Antwort auf den steigenden internationalen Wettbewerbsdruck und den demografischen Wandel in Europa: Nur durch intelligenteres Arbeiten - und damit der besseren Nutzung des Internets - könne der Wohlstand in Europa erhalten bleiben. 
Der Sektor Informations- und Kommunikationstechnologie (IKT) erwirtschaftet bereits etwa fünf Prozent des europäischen Bruttoinlandsprodukts, aber er leistet einen noch größeren Beitrag (nämlich ca. 20 Prozent) zur Steigerung der gesamtwirtschaftlichen Produktivität (Ebd.).

Die Entwicklung von Hochgeschwindigkeitsnetzen hat nach Einschätzung der EU heute die gleiche revolutionäre Wirkung wie einst das Aufkommen der Strom- und Verkehrsnetze. Dennoch kann das Potential der IKT in Europa noch nicht voll genutzt werden, weil ein für das Internet konzipierter Binnenmarkt immer noch erhebliche Lücken aufweist. Die Kommission verweist diesbezüglich auf die noch vergleichsweise schwache Nutzung des Internets durch die Bürger und die im Vergleich zu den USA, Japan und Südkorea geringe Verbreitung von Hochgeschwindigkeits-Glasfasernetzen. Europa bildet noch immer einen Flickenteppich aus nationalen Online-Märkten, die digitalen Dienste in Europa arbeiten nur begrenzt zusammen. Zudem vermindert das Aufkommen von Cyberkriminalität das Vertrauen der Bürger in den Schutz ihrer personenbezogenen Daten. Auch die IKT-bezogenen Forschungs- und Innovationsbemühungen und die digitalen Kompetenzen der Bürger in Europa sind noch zu gering verankert (Ebd.). Vorgeschlagen wird deshalb eine ganze Palette von Maßnahmen, um das wirtschaftlich-soziale Potential von IKT besser zu nutzen. Wie wichtig der Kommission diese Aufgabe ist, zeigt sich daran, dass die digitale Agenda eine der sieben Leitinitiativen der Strategie Europa 2020 ist (siehe Europäische Kommission 2010a). In deren Zentrum stehen die Überwindung der wirtschaftlichen Krise und die Vorbereitung der EU-Wirtschaft auf das nächste Jahrzehnt. Das Strategiepapier identifiziert ein zukunftsgefährdendes europäisches Wachstumsdefizit.

All dies Maßnahmen und Konzepte zeigen, dass die EU die Entwicklung und Förderung des Internets für wichtig hält, insbesondere im Hinblick auf Erhaltung der europäischen Wettbewerbsfähigkeit. Die Strategie der EU ist diesbezüglich eine ,negative Integration', also der Abbau der Schranken für freien und sicheren Internetverkehr und der Ausbau der Infrastruktur, und weniger eine distributive Strategie, dies wäre Abbau der Unterschiede zwischen den europäischen Staaten und vollständige Harmonisierung der nationalen Richtlinien, wie es sonst die Vorgehensweise der EU ist.

\subsubsection{Die Zivilgesellschaft und das Internet}

Das Internet hat auch dazu beigetragen, klassische Strategien und Methoden der politischen Artikulation und Aktion zu beschleunigen und effektiver zu machen. Über das Netz verbreitete Aufrufe zu Protest, Demonstration und auch Rebellion 
zeigen dies deutlich. Darüber sind schon zahlreiche Abhandlungen verfasst worden (Emmer und Bräuer 2010; Busch und Hoffmann 2012). Diese Aspekte brauchen daher kaum vertieft werden. Weniger beleuchtet blieb die Rolle, welche zivilgesellschaftlichen Organisationen bei der Diskussion um und der Reform der eigentlichen Internet Governance gespielt haben und immer noch spielen. Vorab muss darauf hingewiesen werden, dass die sogenannte Zivilgesellschaft theoretisch in enger Definition nur solche Vereine und Verbände umfasst, die weder partei- bzw. staatsgebunden sind noch Profitinteressen verfolgen. Mithin lassen sich unter diese Organisationen (ZGO) mehr als unter die NGOs rubizieren.Diese Kriterien sind allerdings bei weitem nicht bei allen zivilgesellschaftlichen Organisationen gegeben, die sich mit Netzpolitik beschäftigen. Vielmehr sind die Grenzen zwischen bürgerschaftlichem Engagement, der Vertretung wirtschaftlicher Interessen im weiteren Sinne und politischer Einflussnahme in diesem Feld äußerst fließend. Da dies auch in anderen Bereichen beobachtbar ist, viele Länder nur wenige staats-, unternehmens- oder religionsfreie Vereine und Verbände kennen, wird Zivilgesellschaft mitunter auch entsprechend breiter definiert (vgl. Betz 2005).

Außerdem haben sich die Gewichte zwischen staatlichen Organen, den Wirtschaftsverbänden und zivilgesellschaftlichen Organisationen in der Entwicklung des Netzes deutlich verschoben - mit dem Ergebnis, dass der autonome Gestaltungsraum zivilgesellschaftlicher Organisationen deutlich zu schrumpfen begann. In den Anfangsjahren des Netzes war die Zivilgesellschaft dominant, der Einfluss der Regierungen - außer als Finanzgeber - praktisch marginal. Technische Experten definierten die Verfahrensstandards, die Privatwirtschaft und wissenschaftliche Institutionen wendeten sie an und zivilgesellschaftliche Organisationen verteidigten die Interessen der Nutzer. Die technischen Experten arbeiteten in selbstverwalteten Institutionen, die ihre Mitglieder selbst rekrutierten und von ebenfalls staatsfreien Organen (etwa der Internet Society) beaufsichtigt wurden. Lange herrschte in der so gearteten Gemeinschaft der heute etwas naiv erscheinende Optimismus vor, das Internet brauche keinen Staat und dessen Gesetzgebung, selbstverwaltete, virtuelle Gemeinschaften könnten alle Probleme im Konsens lösen (Mueller 2010). Die Entstehungsgeschichte des Netzes und seine "grenzenlose“ Natur motivierten dazu, dass zivilgesellschaftliche Organisationen, die sich mit den Ansprüchen an das Netz und seinem Schutz vor staatlichen Eingriffen befassten, wie Pilze aus dem Boden schossen. Doch das Rad der Geschichte konnte in Bezug auf ihren Einfluss auch dann nicht gänzlich zurückgedreht werden, als sich die Nationalstaaten und die etablierten internationalen Organisationen mit vernehmlichen Reregulierungsabsichten zurückmeldeten. Der Einfluss zivilgesellschaftlicher Organisationen auf ICANN blieb erhalten, sie erhielten Beobachterstatus auf den Weltinformationsgipfeln - samt dem ansonsten auf Gipfeln unüblichen Rederecht -, sie waren mitunter Teil nationaler 
Delegationen und wurden (fast) gleichberechtigte Partner im nachfolgenden Internet Governance Forum.

Es wäre daher trotz des zunehmenden Einflusses staatlicher Instanzen und der wirtschaftlichen Interessen im Netz falsch, bei der Aufzählung der wesentlichen Akteure von Internet Governance die zivilgesellschaftlicher Organisationen $\mathrm{zu}$ übergehen. Leider ist das Schrifttum zu den Zielen, zur Struktur und zum Einfluss dieser Organisationen äußerst dürftig. Die nachfolgenden Ausführungen können diese Lücke nicht füllen, auch nicht entfernt alle zivilgesellschaftlichen Organisationen auflisten, die auf die Internet Governance und ihre Prinzipien Einfluss zu nehmen versuchen.

\subsubsection{Internet Society}

Die politisch bedeutsamste zivilgesellschaftliche Organisation in Bezug auf das Internet ist wohl die Internet Society, die 1992 gegründet wurde und sich für die technische Entwicklung des Internets einsetzt. Sie will die akademische und wissenschaftliche Gemeinschaft über dessen Nutzen und seine Anwendung unterrichten sowie zur Erkundung neuer Anwendungen beitragen. Die Internet Society hat zur Zeit ca. 130 Verbands- und 55.000 persönliche Mitglieder, die Hauptquartiere befinden sich nahe Washington und in Genf. Die Internet Society beherbergt die für die Internetstandards und Ressourcenverwaltung zuständigen Gremien wie die IETF (Internet Engineering Task Force), eine schon genannte Gruppe von Internet-Technikern, und die Internet Assigned Numbers Authority (IANA), welche die Internetressourcen koordiniert; sie hält auch das Copyright an Standards, die von diesen entwickelt wurden. Die Gesellschaft spielte eine nicht unwichtige Rolle auf den Weltinformationsgipfeln, war bei allen Treffen des Internet Governance Forum vertreten und lieferte substantielle Inputs. Sie ist außerdem die Muttergesellschaft des Public Interest Registry, welche die org.-top level domains verwaltet. Die Gesellschaft setzt sich für eine offene, partizipative Entwicklung des Internet zum Nutzen aller Menschen ein; sie atmet also den Geist der früheren Selbstregulierung des Netzes.

\subsubsection{Campaign for Communication Rights in the Information Society}

Ein weiteres sehr wichtiges Netzwerk der internationalen Zivilgesellschaft war und ist die Campaign for Communication Rights in the Information Society - eine Gründung, die den anstehenden Weltinformationsgipfel nutzte, um die internationale Öffentlichkeit auf linksprogressive Kommunikationsanliegen aufmerksam zu machen. Sie hat auf diesem Gipfel einen wesentlichen inhaltlichen Input 
geliefert, blieb aber institutionell fragil; denn sie war stets in schwieriger finanzieller Situation und strebte letztlich zu viel auf einmal an (Mathiason 2009).

\subsubsection{Association for Progressive Communications (APC)}

Die Association for Progressive Communications (APC) ist ein 1990 gegründetes internationales Netzwerk von Organisationen um Gruppen und einzelnen Intellektuellen, um Internetanwendungen zur Verfügung für die Förderung des Friedens, der Menschenrechte und den Schutz der Umwelt, insbesondere auch in ärmeren Ländern, zu stellen. Etwa 50 Netzwerke sind Mitglieder von APC, die meisten davon aus Entwicklungsländern. APC gibt zusammen mit einer niederländischen Organisation ein Jahrbuch heraus, das Fortschritte auf dem Weg zu einer inklusiven Weltinformationsgesellschaft dokumentiert und entwickelt Apps für die Websites von NGOs, um deren Sichtbarkeit zu erhöhen. Die APC wies äußerst prominente Leitfiguren der Kommunikations- und Internetforschung auf, neigte vielleicht auch daher zu Abspaltungen und Mutationen. Aus ihr gingen noch in den 1990er Jahren die Peoples Communication Charter, die Platform for Cooperation on Democratization and Communication sowie Voices 21 hervor.

\subsubsection{Free Software Foundation}

Derselben Aufgabe widmet sich die Free Software Foundation, die 1985 in Boston gegründet wurde. Sie hat bis Mitte der 1990er Jahre ihre Mittel dazu verwandt, Programmierer für die Entwicklung freier Software anzustellen. Die FSF bemüht sich seither um allgemeine Beratung und Aufklärung rund um freie Software und hat ein Verzeichnis freier Software aufgelegt. Die Stiftung wendet sich gegen zu unternehmensfreundliche Regelungen des Urheberrechts.

\subsubsection{Weitere NGOs}

Schon auf dem ersten Weltinformationsgipfel waren Tausende von NGOs aktiv, davon immerhin 700 mit regelmäßigen Aktivitäten. Diese schlossen sich auf dem Gipfel zu verschiedenen Netzwerken zusammen, die etwa die Kommunikationstechnologien in den Dienst der wirtschaftlichen und sozialen Entwicklung stellen wollten (darunter das World Press Freedom Committee, die Reporter ohne Grenzen, Amnesty International). Außerdem wollen sie die Menschenrechte und Meinungsfreiheit über das Netz fördern, sich mit der Weiterentwicklung von ICANN befassen, das Copyright entschlacken, freie Software und die Informationsfreiheit fördern (z. B. die Free Software Foundation, Knowledge Ecology International). Die APC wurde zum zentralen Knotenpunkt dieser Netzwerke, weil sie über professionelle Stäbe in London und Johannesburg verfügt und alle Themen abdecken kann (Mueller 2010). 
Auch in Deutschland gibt es Dutzende von zivilgesellschaftlichen Organisationen und Wirtschaftsverbänden, die sich der Internetpolitik widmen. Sie wirken auch etwa an der Enquete-Kommission des Bundestages zu Internet und digitale Gesellschaft mit. Es würde den Rahmen dieses Kapitels sprengen, alle aufzuzählen. Die meisten internationalen ZGOs haben ihre Ableger in der Bundesrepublik, mit einem zum Teil beachtlichen Mitgliederstand. Daneben gibt es aber auch zahlreiche nationale Initiativen und Verbände:

\subsubsection{Wirtschaftsverbände}

Bei den Wirtschaftsverbänden weisen der Bundesverband Digitale Wirtschaft und der Bundesverband Informationswirtschaft, Telekommunikation und neue Medien (BITKOM) besondere Internet-Kompetenz auf. Der erst genannte Verband wurde 1995 gegründet, hat ca. 600 Mitglieder aus den Bereichen E-Commerce, Online-Vermarktung, Onlinemedia- und Internetagenturen sowie Social Media. Sitz des Vereins ist Düsseldorf. Er veranstaltet seit 2009 eine einschlägige Messe (in Köln) und einen Online-Wettbewerb. Zudem veröffentlicht er Daten und Prognosen zum Deutschen Online-Werbemarkt und publiziert ein Ranking der Internetagenturen. BITKOM ist der Branchenverband der deutschen Informationsund Telekommunikationsbranche. Der Verband wurde 1999 in Berlin von einschlägigen Fachverbänden gegründet. Ihm gehören alle Großen der Branche an, er vertritt zur Zeit etwa 1.700 Unternehmen. BITKOM setzt sich dafür ein, gute wirtschaftliche und rechtliche Rahmenbedingungen für die Informations- und Kommunikationsbranche in Deutschland und der EU zu fördern. Der Verband hat auch immer wieder Kritik an der Technologiepolitik der Bundesregierung geübt, ist publizistisch sehr aktiv und veranstaltet jährlich einen BITKOM Trendkongress, an dem hochrangige Vertreter aus Politik und Wirtschaft teilnehmen.

Etwas kleiner (ca. 400 Mitglieder) als die beiden genannten Vereine ist der Verband der deutschen Internetwirtschaft (Eco), der 1995 in Bonn gegründet wurde. Er ist politisch aber recht aktiv, hat sich maßgeblich in die Gestaltung des deutschen Telekommunikationsgesetzes eingebracht. Mit seinem eco award werden jährlich besonders innovative und erfolgreiche IT-Unternehmen ausgezeichnet.

\subsubsection{Politische Vereine}

Es gibt auch etliche politiknahe Internet-Vereine: Am offenkundigsten ist dies bei den parteieigenen Assoziationen. So gründete etwa die CDU im Frühjahr 2012 mit $\mathrm{CNetz}$ einen eigenen Verein, in dem auch die CDU-Vertreter in der EnqueteKommission des Deutschen Bundestages Mitglieder sind. Der Verein, der als ein Ziel definierte, das Internet nicht nur den Linken zu überlassen (Der Tagesspiegel 4. April 2012), steckt noch in den Anfängen. Der entsprechende Ableger der 
SPD (D64) ging schon Ende 2011 an den Start, der Ableger der Grünen (Digiges) bereits im April 2011. Diese Vereinigungen verdanken ihre Entstehung nicht unwesentlich dem politischen Erfolg der Piraten; sie möchten aber auch progressiv in die Parteien (etwa zum Thema Datenspeicherung) hineinwirken.

Eine regierungsnahe Initiative ist der Verein Deutschland sicher im Netz e. V., der sich der Aufgabe widmet, Nutzer zum Thema Internetsicherheit zu beraten. Er wurde 2005 gegründet und steht unter der Schirmherrschaft des deutschen Wirtschaftsministeriums. Er veröffentlicht ein Sicherheitsbarometer, das vor Risiken im Netz warnt, und er zählt zu seinen Mitgliedern große Telekom- und Internetunternehmen sowie verschiedene NGOs.

Die Initiative D21 e. V. ist Deutschlands größte Partnerschaft von Politik und Wirtschaft zur Ausgestaltung der Informationsgesellschaft. Die Initiative wurde 1999 in Berlin unter Federführung des damaligen Chefs von IBM Deutschland gegründet und umfasst ein Netzwerk von 200 Mitgliedsunternehmen und -institutionen sowie politischen Parteien. D21 veröffentlicht jährlich einen Atlas, der die Verbreitung der Informationstechnologie und der IT-Kompetenzen in Deutschland anzeigt.

Das Bündnis Freiheit statt Angst ist im Wesentlichen durch die Abhaltung von jährlichen Demonstrationen aktiv, setzt sich für den Datenschutz und gegen Überwachung ein und wird von einer breiten Palette politischer Parteien, Menschenrechtsorganisationen und Berufsverbänden unterstützt.

Der Förderverein für eine Freie Informationelle Infrastruktur e. V., gegründet 1999 und mit Sitz in München, hat über 1000 Mitglieder und widmet sich der Schaffung und Verwendung von frei implementierbaren Standards; er unterstützt Softwareanwender in ihrem Bemühen um freie Software und Open Content, wird jedoch auch von Softwareentwicklern gefördert. Der Verein weist eine offene Partizipationsstruktur auf und will eine Unterstützerbasis von mehr als 50.000 Personen haben. Er arbeitet eng mit Datenschutzorganisationen zusammen. Die Hauptaktivität des Vereins richtet sich auf die Freiheit des Programmierens, die er durch die EU-Richtlinie zur Patentierbarkeit von Software gefährdet sah. Diese Richtlinie wurde vom Europäischen Parlament abgelehnt. Gegen eine überarbeitete, wiederum wirtschaftsfreundliche Regelung hat der Verein in Kooperation mit anderen eine Petition eingebracht.

Kleinere Initiativen sind etwa die Digitale Gesellschaft e. V., ein im Jahr 2010 gegründeter Verein, der sich für die Bürgerrechte und den Verbraucherschutz im Netz einsetzt. Ferner will das Netzwerk Neue Medien den Eintritt Deutschlands in die Informationsgesellschaft politisch begleiten; deshalb arbeitete es anfangs stark den "Grünen“ zusammen.

Auch klassische Vereine für den Datenschutz sind netzpolitisch aktiv. Dazu gehört in Deutschland v. a. die Gesellschaft für Datenschutz und Datensicherheit e. V., 
die schon 1977 gegründet wurde und über 2200 Mitglieder hat. Sie tritt für einen sinnvollen, vertretbaren Datenschutz ein und unterstützt Datenschutzbeauftragte und datenverarbeitende Stellen dabei, den Datenschutz und die Datensicherheit umzusetzen. Kleiner ist die Deutsche Vereinigung für Datenschutz e. V., deren Ziel der Schutz persönlichen Daten ist und die diesbezüglich mit anderen NGOs zusammenarbeitet und mit diesen zusammen die jährliche Verleihung des deutschen Big Brother Awards organisiert.

Nicht darf fehlen in dieser Aufzählung der Chaos Computer Club (CCC), ein Verein, in dem sich Hacker zusammengeschlossen haben. Der CCC wurde 1981 mit Sitz in Hamburg gegründet; er fordert ein neues Menschenrecht auf weltweite, ungehinderte Kommunikation. Die Mitgliedschaft steht jedem offen, der sich mit den Zielen des Vereins identifizieren kann. Der Club hat zur Zeit ca. 3000 Mitglieder, die sich unter anderem jährlich zum Chaos Communication Congress treffen. Der CCC gibt als Zeitschrift Die Datenschleuder heraus, zusätzlich publizierte er die Hackerbibel und betreut diverse Radiosendungen mit Hackerinhalten. Der Verein machte auch mit spektakulären Angriffen auf Netze von Unternehmen aufmerksam, mit denen deren Sicherheitsmängel demonstriert werden sollten. Der CCC wurde daher bei der Schaffung des deutschen Datenschutzgesetzes immer wieder zu Rate gezogen. 